\title{
Quantitative Support for Borowski's Theory of Gravitation
}

\author{
Michael A. Persinger \\ Laurentian University, Sudbury, Ontario, Canada \\ E-mail address: mpersinger@laurentian.ca
}

\begin{abstract}
The Borowski Theory of Gravitation (BTG) indicates that movements of mass such as planets through space are determined by differential pressures from dark matter. One of the consequences of the final epoch is that there would be no matter but only distance. Quantitative solutions indicate that the tensor to set universal average dark matter pressure equal to $\mathrm{G}$, the gravitational constant, would require that the terminal length would be $\sim 2.2 \cdot 10^{69} \mathrm{~m}$ or effectively identical to current estimates of energy equivalence of the universal mass. For the earth's orbit the force from the dark pressure is the same order of magnitude as the force associated with the product of the planet's mass and background free oscillations whose origins are still ambiguous. The convergences of solutions suggest that the BTG may reveal alternative interpretations and mechanisms for the role of gravitation in planetary motion.
\end{abstract}

Keywords: Dark matter; gravitation; quantitative solutions; final epoch; planetary motions; spaceenergy relations

\section{INTRODUCTION}

Despite the multiple and often very elegant mathematical descriptions that describe gravitation the mechanisms that determine the interaction between masses and their movements through the universe can be considered ambiguous. Borowski [1,2] has presented a theory predicated on: 1) parsimony, and, 2) the concept that motions of planets around the Sun, for example, are mediated by differences in dark matter pressures. The whole planetary system is assumed to move through an intrinsic solvent that is considered dark matter. The pressure of dark matter is greater the more distant the point from an aggregated mass such as a star or a planet and less the more proximal to the material mass. There is both conceptual and quantitative support for the Borowski theory.

\section{THE SIMPLICITY OF THE UNIVERSAL SET}

One of the major impedances involved with the conceptualization of forces and energies is the implicit inclusion of processes or dynamics rather than unit or static increments. All phenomena that involve processes require at least two increments of time $(\Delta t)$ to be discerned. There is a general relationship between the $\Delta \mathrm{t}$ to discern or perceive a phenomenon and the 
increment of space $(\Delta s)$ within which the phenomenon exists or is defined. For example, the optimal discrimination of the activities of atoms which are measured in picometer space involves picoseconds rather than attoseconds or megaseconds. The optimal discrimination of geophysical patterns, that occur in megameter space involve increments of $10^{6} \mathrm{~s}$ rather than $10^{-3} \mathrm{~s}$ or $10^{10} \mathrm{~s}$.

If the $\Delta$ ts are less or greater than the optimal increment, the phenomenon is fragmented into uncorrelated components or becomes a variable aggregate of summed or integrated units. The logical boundary to this approach is that at the largest $\Delta \mathrm{s}$ there will be only one $\Delta \mathrm{t}$ and hence no process. The largest increment of space is the universe. This means when the whole set, the universe, is considered there are no dynamics. The simplicity that results from this static set allows the potential to apply simpler and more fundamental relationships. From this perspective there is minimum requirement for intricate and extrapolative mathematical descriptions of local space-time geometries.

For example the duration of the entire static set, the universe, could be estimated by the $\mathrm{G} \delta$ where $\mathrm{G}$ is the gravitational constant $\left(6.67 \cdot 10^{-11} \mathrm{~m}^{3} \cdot \mathrm{kg}^{-1} \cdot \mathrm{s}^{-2}\right)$ and $\delta$ is assumed to be the mean density of 1 proton per cubic meter. The resulting value is $11.1 \cdot 10^{-38} \mathrm{~s}^{-2}$. The inverse is $\sim .09 \cdot 10^{38} \mathrm{~s}^{2}$. The square root value is $\sim 3 \cdot 10^{18} \mathrm{~s}$ or the equivalent of 90 billion years [3]. This value is similar to the estimates by Hoffman et al [4] who estimated that in an open cold matter (OCDM) model the boundary for the final epoch would be 89.2 billion years. This means that the present universe is only about $14 \%$ of that value. Ordinary baryonic matter accounts for $10 \%$ to $20 \%$ of the masses of major galactic clusters; the remaining has been attributed to dark matter and energy [5].

Persinger [3] has suggested that dark matter and energy are entities that are yet to occur but display their effect indirectly in a manner similar to Mach's principle for space but applied to time within a four-dimensional system. If pressure from dark matter contributes to motion, particularly acceleration, then the gradual diminishment of dark matter as the universe approaches the final epoch should be associated with slower and slower expansion, that is, it decelerates [2]. Such an approach and interpretation becomes more easily understood when the simplicity of the entire set is considered.

\section{DISAPPEARANCE OF MASS AND ONLY SPACE REMAINS}

According to Borowski as the universe approaches infinity the total mass of the universe approaches zero. There is quantitative support for this concept. Persinger [6] showed that the relationship between universal pressure, calculated as the product of average density $\left(1.67 \cdot 10^{-27} \mathrm{~kg} \cdot \mathrm{m}^{-3}\right.$ or 1 proton per $\left.\mathrm{m}^{3}\right)$ and the velocity of light, c, squared $\left(9 \cdot 10^{16} \mathrm{~m}^{2} \cdot \mathrm{s}^{-2}\right)$ or $1.50 \cdot 10^{-10} \mathrm{~Pa}$, and the gravitational constant, $\mathrm{G}$, could be accomplished by multiplying the former by $\mathrm{m}^{4} \cdot \mathrm{kg}^{-2}$. Assuming the typical distance across the universe was $\sim 10^{26} \mathrm{~m}$ this meant that the transform was $10^{104} \mathrm{~m}^{4}$ while the mass was $10^{104} \mathrm{~kg}^{-2}$ in order to maintain unity. Consequently the mass of the universe was $2.4 \cdot 10^{52} \mathrm{~kg}$ when the coefficients were considered. This value was remarkably similar to empirical estimates based upon $\sim 2 \cdot 10^{30} \mathrm{~kg}$ per star (assuming the sun's mass), $10^{11}$ stars in the galaxy and $10^{11}$ galaxies in the universe.

To be consistent with Borowski's model as distance increases mass must decrease. Ultimately as mass approaches 1 the distance approaches $10^{208} \mathrm{~m}^{4}$ or, when the cube root is taken for volume, the linear distance becomes $2.2 \cdot 10^{69} \mathrm{~m}$. The exactness of this value is relevant. The rest energy $E_{0}$ or intertial mass of a system is equal to the mass $M_{0}$ which can be considered a characteristic of the systems' gravitational field. One classical means of relating energy to $\mathrm{G}$ and $\mathrm{M}$ is the equation: 


$$
8 \pi \mathrm{M}^{2} \mathrm{GR}^{-1}=\mathrm{Mc}^{2}
$$

where $\mathrm{R}$ is radius derived from:

$$
f=8 \pi \mathrm{Gc}^{-2}
$$

such that the resulting $1.86 \cdot 10^{-26} \mathrm{~m} \cdot \mathrm{kg}^{-1}$ multiplied by the mass of $2.4 \cdot 10^{52} \mathrm{~kg}$ results in $\mathrm{R}=$ $4.4 \cdot 10^{26} \mathrm{~m}$. With these parameters the resulting total energy within the universe would be $2.2 \cdot 10^{69} \mathrm{~J}$ [7]. This is the same value predicted for the maximum distance when matter disappears according to the theory.

\section{THE DARK MATTER SOLVENT}

According to Borowski's theory of gravity the differential densities of dark matter drive the subtle changes in planetary motion. If dark matter pressures are assumed to be similar to the intrinsic pressure derived from $\delta \mathrm{c}^{2}$ or $1.50 \cdot 10^{-10} \mathrm{~Pa}$, then convergences occur that support the contention. The surface area of the planetary orbit of the earth $\left(\mathrm{r} \sim 1.5 \cdot 10^{11} \mathrm{~m}\right)$, for example, is $2.83 \cdot 10^{23} \mathrm{~m}^{2}$. So the dynamic pressure induced "force" from this origin would be $4.25 \cdot 10^{13} \mathrm{~N}$.

The motion of a mass through the dark matter pressure would be expected to elicit an intrinsic resonance or free oscillation around the mass that would be reflected as a relatively small magnitude in units of acceleration. Free oscillations or fundamental spheroidal modes within the earth occupy a relatively wide band of perturbations between $2 \mathrm{mHz}$ and $7 \mathrm{mHz}$ whose general peak to peak amplitudes are $\sim 0.5 \mathrm{nGal}$ where $1 \mathrm{nGa}=10^{-11} \mathrm{~m} \cdot \mathrm{s}^{-2}[8,9]$. The origins of these oscillations are not clear but have been attributed to some process within the boundary between the earth's surface and the atmosphere [9].

When this acceleratory term is multiplied by the mass of the earth, $5.98 \cdot 10^{24} \mathrm{~kg}$, the "spheroidal" force emerges as $3 \cdot 10^{13} \mathrm{~N}$. The similar order of magnitude between the force from putative "dark matter" pressure over the surface of the earth's orbital shell and the force associated with the earth's mass and "free oscillations" would be expected if they shared a dynamic source. In the BTG approach the velocity of a planet moving in an elliptical orbit is minimal the furthest from the sun because the dark pressure is potentially the highest. Intuitively this should produce slight increases in the amplitude of the planet's free oscillations because of the slightly higher dark matter pressures. Interestingly there is an annual variation in amplitude with a peak when the earth is most distal $(0.6 \mathrm{nGal})$ compared to most proximal $(0.4 \mathrm{nGal})$.

\section{EQUIVALENCE OF ENERGIES}

If the force from the assumed pressure from dark matter is applied over the mean distance of the earth's orbital radius $\left(1.5 \cdot 10^{11} \mathrm{~m}\right)$ the resulting energy is $6.4 \cdot 10^{24} \mathrm{~J}$. On the other hand the dynamic "dark matter" pressure within the volume of the earth's orbit from the average distance would be the product of $\left(1.42 \cdot 10^{34} \mathrm{~m}^{3}\right) \cdot\left(1.67 \cdot 10^{-27} \mathrm{~kg} \cdot \mathrm{m}^{-3}\right)$ or $2.37 \cdot 10^{7} \mathrm{~kg}$. The energy equivalence of this mass is $2.1 \cdot 10^{24} \mathrm{~J}$. Thus the energy associated with moving 
through the dark matter fields approach equity with the energy equivalence of the dark matter within the volume occupied by the earth's orbit.

\section{SOME EMERGENT FEATURES}

Any new approach should converge with established measurements. For example the standard earth model for mean moment of inertia of the entire earth is $8.02 \cdot 10^{37} \mathrm{~kg} \cdot \mathrm{m}^{2}$. When divided by the energy equivalence of the dark matter mass within the orbital volume $\left(2.1 \cdot 10^{24}\right.$ $\mathrm{J})$, the duration (after $\sqrt{\mathrm{s}^{2}}$ ) is $0.61 \cdot 10^{7} \mathrm{~s}$ which is within the same order of magnitude as the earth's orbital latency around the sun. When this procedure is applied to all of the planets, the discrepancy between the actual orbital times (in years) and the predicted orbital durations derived from their moments of inertia divided by the energy equivalences of the assumed dark matter density within the volumes of spaces that comprise their orbits is least for Mercury and Venus, those closest to the largest mass in the solar system, and Jupiter, the second largest mass in the solar system.

For these three planets the concordance between the predicted versus observed orbital durations is $>50 \%$. For Earth and Mars, the predicted values are $20 \%$ and $2 \%$ of the observed values, respectively, while for Saturn, Uranus, and Neptune, the concordance is only $3 \%, 0.1 \%$ and $0.01 \%$, respectively. As predicted by Borowski's gravitational theory the discrepancy should occur because the density of dark matter pressure increases the further away from large masses. Hence the quantitative density must be greater for the observed and predicted values to converge. Near large masses the pressure would be approaching a lowest value.

\section{CONCLUSIONS}

Explanations of the processes by which the phenomenon of gravitation occurs may require an alterative approach that incorporates differences in dark matter pressure. Borowski's qualitative predictions concerning the final condition of the universe as ultimately space and no mass as well as the differential motion of planets due to changing densities of dark matter pressures within distances from larger masses are supported by quantitative estimates. Systematic application of this theory may reveal more concise explanations and unexpected properties in physical cosmology.

\section{References}

[1] T. Borowski, International Letters of Chemistry, Physics and Astronomy 1 (2012) 1-5.

[2] T. Borowski, International Letters of Chemistry, Physics and Astronomy 11 (2013) 44-53.

[3] M. A. Persinger, International Journal of Astronomy and Astrophysics 3 (2012) 125-128.

[4] Y. Hoffman, O. Lahav, G. Yepes, Y. Dover, Journal of Cosmology and Astroparticle Physics 37 (2007) 1-16.

[5] T. Ponman, Science 414 (2001) 402-404.

[6] M. A. Persinger, Journal of Physics, Astrophysics and Physical Cosmology 3 (2009) 1-3. 
[7] M. A. Persinger, International Letters of Chemistry, Physics and Astronomy 8 (2013) 8-19.

[8] T. Tanimoto, J. Ulm, K. Nishida, N. Kobayashi, Geophysical Letters 25 (1998) 1553-1563.

[9] K. Nishida, N. Kobayashi, Y. Fukao, Science 287 (2000) 2244-2246. 